\title{
Oral Cancer Awareness among Dentists in Kuwait
}

\author{
Bobby K. Joseph ${ }^{a}$ Devipriya B. Sundaram ${ }^{a}$ Prem Sharma $^{b}$ \\ a Department of Diagnostic Sciences, Faculty of Dentistry, and b Health Sciences Center, Kuwait University, \\ Jabriya, Kuwait
}

\section{Key Words}

Oral cancer $\cdot$ Dentists $\cdot$ Kuwait

\begin{abstract}
Objectives: The aim of this study was to assess oral cancer awareness among dentists in Kuwait. Subjects and Methods: A cross-sectional survey was conducted among 200 dentists working at the Ministry of Health Dental Centers and Kuwait University Dental Center using a structured questionnaire. Dentists' knowledge about risk factors of oral cancer and about diagnostic concepts, current practices and opinions, preferred point of referral as well as interest in continuing education were assessed and the responses were analyzed. Results: Of the 200 dentists surveyed, 153 responded (76.5\% response rate). The mean knowledge score of the respondents was $20.6 \pm 4.0$ out of a total score of 30 . Thirty-five (22.9\%) dentists had consistently high knowledge scores for both risk factors and diagnostic concepts. Of the 153 dentists, 132 (86.3\%) were interested in obtaining further information about oral cancer. Conclusion: This study highlighted the need for improved knowledge and education of dental practitioners on oral cancer.
\end{abstract}

Copyright $\odot 2011$ S. Karger AG, Basel
(C) 2011 S. Karger AG, Basel

1011-7571/12/0212-0164\$38.00/0

Fax +4161306 1234

E-Mail karger@karger.ch

www.karger.com

\section{Introduction}

The mouth and oropharynx are among the ten most common sites affected by cancer worldwide, but global incidence varies widely [1]. Globally, the age-standardized (world) incidence rate (per 100,000) for lip/oral cavity cancer is 3.8 [2]. In Kuwait, the incidence rate is 1.4 and the mortality rate is 0.6 . The incidence rate is slightly higher in women $(1.8$ per 100,000$)$ than in men $(1.1$ per 100,000) [2].

Despite advances in therapeutic management and increased understanding of the molecular basis of the disease, survival rates have not improved in the last few decades. The 5 -year survival rates exceed $50 \%$ in only the best treatment centers [1]. However, early diagnosis of oral cancer greatly increases the probability of cure with minimum impairment and deformity [3].

A dental practitioner's negative attitude and low level of knowledge are considered to be factors that contribute to the delayed or inadequate detection of the early stages of oral cancer [4]. Consistently performing thorough oral cancer screening examination for all patients (including high-risk areas like tongue and floor of mouth), paying careful attention to suspicious red, white and ulcerative mucosal lesions and being aware of patients' high-risk tobacco and alcohol use offer the best potential for dentists to detect oral cancer at an early stage [5-10].

Bobby K. Joseph

Department of Diagnostic Sciences, Faculty of Dentistry, Kuwait University PO Box 24923

Safat 13110 (Kuwait)

Tel. +965 2498 6694, E-Mail bobby@ hsc.edu.kw 
Table 1. Background characteristics of respondents $(n=153)$

\begin{tabular}{lr}
\hline Background characteristic & $\mathrm{n}(\%)$ \\
\hline $\begin{array}{l}\text { Gender } \\
\quad \text { Male }\end{array}$ & $118(77.1)$ \\
$\quad$ Female & $35(22.9)$ \\
Age group & \\
$\quad<40$ years & $86(60.6)$ \\
$\quad \geq 40$ years & $56(39.4)$ \\
Nationality & \\
$\quad$ Kuwaiti & $48(31.4)$ \\
$\quad$ Non-Kuwaiti & $105(68.6)$ \\
Time since graduation & \\
$\quad$ More than 15 years (before 1994) & $63(44.4)$ \\
$\quad$ Within 15 years (1994-2009) & $79(55.6)$ \\
\hline
\end{tabular}

Several studies in the USA $[5,8,10,11]$, Canada [6], Europe [7, 9, 12-14] and Saudi Arabia [15] have assessed dentists' knowledge, opinions and practices regarding oral cancer. These studies showed the need to improve the knowledge on preventing and detecting oral cancer. To date, there has been no published detailed analysis assessing dentists' oral cancer knowledge, opinions and practices in Kuwait.

Dentists working in Kuwait received formal education and training in prevention and detection of oral cancer during their undergraduate studies in their respective dental schools. However, there are no specific courses currently available in Kuwait for the dentists working in various sectors. Therefore, assessing their knowledge and practices would provide baseline information about their levels of knowledge and need for further improvement. Hence, the aim of this study was to assess the knowledge, diagnostic concepts, practices and opinions of dentists in Kuwait regarding oral cancer prevention and early detection.

\section{Subjects and Methods}

Kuwait offers free dental services through government dental centers and polyclinics spread over six governorates. The Kuwait University Dental Center also provides free dental care to patients from all over the country. According to available data, a total of 961 dentists work in the government hospitals while 35 dentists work in the Kuwait University Dental Center. A cross-sectional survey was conducted among these dentists with the approval of the Institutional Ethics Committee as well as permission to conduct the survey in the Ministry of Health Dental Centers.

To arrive at a representative sample, it was estimated that the sampling of about 199 of this dentist population of 996 would give the study a power of $80 \%$ with $95 \%$ confidence interval while keeping a 6\% margin of error. Accordingly, a 4-page English questionnaire was distributed anonymously to 200 dentists proficient in the English language, who work in the various governorates as well as the Kuwait University Dental Center. The questionnaire included previously validated terms as well as items specifically designed for this survey $[5,11]$.

The dentists' knowledge of oral cancer was tested under two main categories: (a) knowledge of risk factors, and (b) knowledge of clinical diagnostic concepts. The answers were predominantly in the 3-point scale of 'yes', 'no', 'don't know' and a few were in the 'multiple choice' format. A knowledge score was calculated based on the correct responses to the 30 questions with a score of ' 1 ' for each correct answer. Depending on the number of correct responses, knowledge indices were created for both risk factors and diagnostic concepts using previously developed scales $[5,6]$.

In addition, current practices and opinions regarding oral cancer as well as their interest in continuing education (CE) were explored. Demographic characteristics and information about education of oral cancer received during their period of study at the respective schools of dentistry were also included in the questionnaire.

The data management and statistical analysis were performed using the statistical software SPSS 16.0. The mean knowledge scores were compared using Student's t test. The $\chi^{2}$ test was applied to find an association between background characteristics and the knowledge scores. Nonparametric Spearman's $(\rho)$ test was used to establish any correlation between the knowledge of risk factors and the knowledge of diagnostic concepts. A two-sided probability ( $p$ value) $<0.05$ was considered statistically significant.

\section{Results}

From a total of 200 questionnaires distributed, 153 dentists returned completed questionnaires giving a response rate of $76.5 \%$. The background characteristics of the respondents are presented in table 1 . The percent distribution of correct responses to questions on knowledge of risk factors ranged from 8.8 to $100.0 \%$ as shown in figure 1 . The mean $\pm \mathrm{SD}$ correct risk factor knowledge score was $8.8 \pm 2.3$ (range 2-14) based on 15 items.

The percentage of correct responses to the questions on knowledge of diagnostic concepts is shown in figure 2. The mean $\pm \mathrm{SD}$ correct diagnostic knowledge score was $11.8 \pm 2.4$ (range $2-13$ ) based on 15 items. The percent distribution of correct responses ranged from 50.4 to $98.0 \%$.

The distribution of dentists according to levels of knowledge about risk factors and clinical diagnosis is presented in table 2 . Thirty-five (22.9\%) dentists had consistently high scores on both indices while 13 (8.5\%) scored low on both indices. Spearman's $\rho$ showed a significant association between dentists' knowledge of risk factors and knowledge of clinical diagnostic concepts $(\mathrm{p}<0.001)$. 


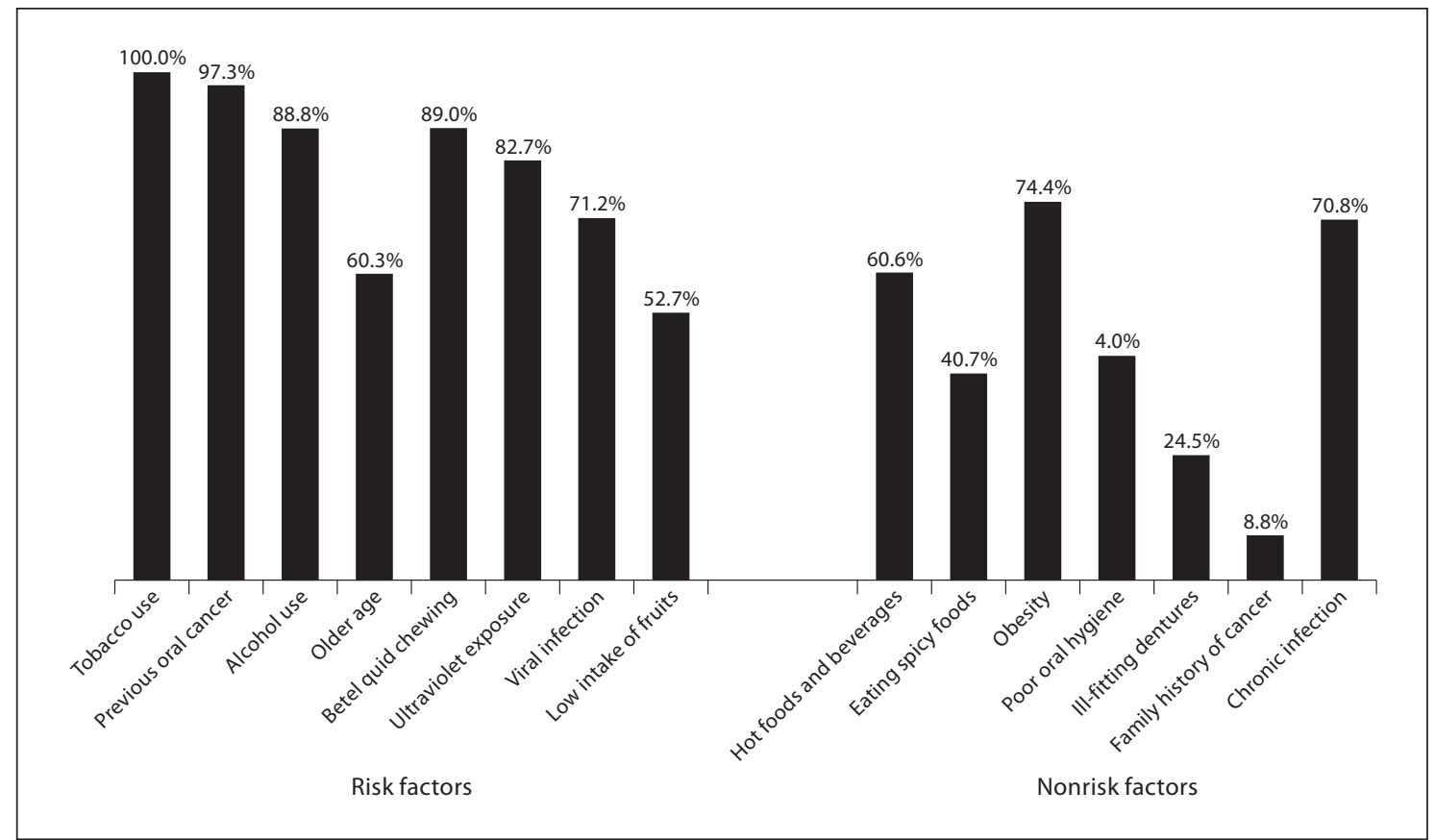

Fig. 1. Percentage of dentists who identified the risk factors of oral cancer.

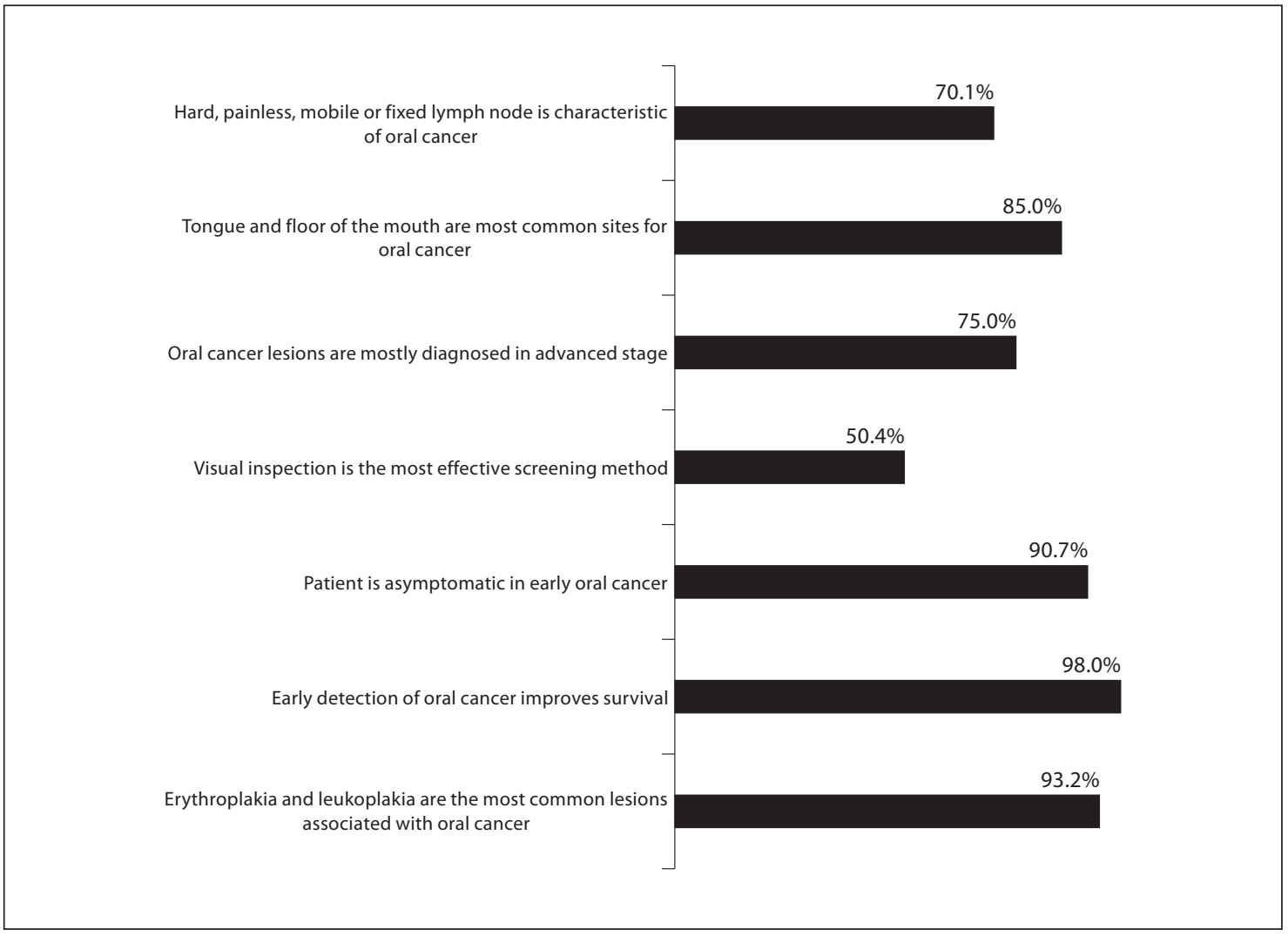

Fig. 2. Percentage of dentists who gave correct responses to diagnostic knowledge items. 
Table 2. Distribution of dentists by levels of knowledge about risk factors and clinical diagnosis of oral cancer

\begin{tabular}{|c|c|c|c|c|}
\hline \multirow[t]{2}{*}{ Knowledge of risk factors ${ }^{a}$} & \multicolumn{4}{|c|}{ Knowledge of clinical diagnostic concepts ${ }^{\mathrm{b}}(\mathrm{n}=153), \mathrm{n}(\%)$} \\
\hline & $\begin{array}{l}\text { low score } \\
\text { (0-8 items) }\end{array}$ & $\begin{array}{l}\text { medium score } \\
\text { ( } 9 \text { or } 10 \text { items) }\end{array}$ & $\begin{array}{l}\text { high score } \\
\text { (11-15 items) }\end{array}$ & all dentists \\
\hline Low score ( $0-8$ items $)$ & $13(8.5)$ & $13(8.5)$ & $26(17.0)$ & $52(34.0)$ \\
\hline Medium score ( 9 or 10 items) & $4(2.6)$ & $5(3.3)$ & $55(35.9)$ & $64(41.8)$ \\
\hline High score (11-15 items) & $0(0.0)$ & $2(1.3)$ & $35(22.9)$ & $37(24.2)$ \\
\hline All dentists & $17(11.1)$ & $20(13.1)$ & $116(75.8)$ & $153(100.0)$ \\
\hline
\end{tabular}

a Total of 15 risk factor items. ${ }^{b}$ Total of 15 diagnostic items; $\rho=0.439(\mathrm{p}<0.001)$.

Table 3. Bivariate associations between general characteristics and knowledge of risk factors and diagnostic concepts

\begin{tabular}{|c|c|c|c|c|c|c|c|c|}
\hline \multirow{2}{*}{$\begin{array}{l}\text { General } \\
\text { characteristic }\end{array}$} & \multicolumn{4}{|c|}{ Risk factor knowledge, n (\%) } & \multicolumn{4}{|c|}{ Diagnostic knowledge, n (\%) } \\
\hline & low score & medium score & high score & $\mathrm{p}$ value & low score & medium score & high score & $\mathrm{p}$ value \\
\hline \multicolumn{9}{|l|}{ Gender } \\
\hline Male & $43(36.4)$ & $48(40.7)$ & $27(22.9)$ & \multirow[t]{2}{*}{0.49} & $14(11.9)$ & $15(12.7)$ & $89(75.4)$ & \multirow[t]{2}{*}{0.85} \\
\hline Female & $9(25.7)$ & $16(45.7)$ & $10(28.6)$ & & $3(8.6)$ & $5(14.3)$ & $27(77.1)$ & \\
\hline \multicolumn{9}{|l|}{ Age Group } \\
\hline$<40$ years & $23(26.7)$ & $38(44.2)$ & $25(29.1)$ & \multirow[t]{2}{*}{0.08} & $7(8.1)$ & $12(14)$ & $67(77.9)$ & \multirow[t]{2}{*}{0.34} \\
\hline$\geq 40$ years & $25(44.6)$ & $20(35.7)$ & $11(19.6)$ & & $9(16.1)$ & $7(12.5)$ & $40(71.4)$ & \\
\hline \multicolumn{9}{|l|}{ Nationality } \\
\hline Kuwaiti & $7(14.6)$ & $26(54.2)$ & $15(31.2)$ & \multirow[t]{2}{*}{$<0.001$} & $5(10.4)$ & $5(10.4)$ & $38(79.2)$ & \multirow[t]{2}{*}{0.77} \\
\hline Non-Kuwaiti & $45(42.9)$ & $38(36.2)$ & $21(22)$ & & $12(11.4)$ & $15(14.3)$ & $78(74.3)$ & \\
\hline \multicolumn{9}{|l|}{ Graduation } \\
\hline Before 15 years & $25(39.7)$ & $28(44.4)$ & $10(15.9)$ & \multirow[t]{2}{*}{0.08} & $6(9.5)$ & $10(15.9)$ & $47(74.6)$ & \multirow[t]{2}{*}{0.86} \\
\hline Within 15 years & $22(27.8)$ & $32(40.5)$ & $25(31.6)$ & & $8(10.1)$ & $10(12.7)$ & $61(77.2)$ & \\
\hline
\end{tabular}

The bivariate associations with risk factor and diagnostic knowledge are displayed in table 3 . Kuwaitis scored significantly higher in the knowledge of risk factors than the non-Kuwaiti dentists $(\mathrm{p}<0.01)$. A lower percentage $(\mathrm{n}=27 ; 27.8 \%)$ of younger dentists ( $<40$ years) scored low in risk factor knowledge compared to dentists in the older age group ( $\geq 40$ years) ( $n=25 ; 44.6 \%)$. A higher percentage of dentists who graduated within 15 years $(n=27$; $30 \%)$ received high scores in risk factor knowledge than dentists who graduated 15 years ago.

The current practice of dentists regarding oral cancer is shown in figure 3. Ninety-five (62.9\%) dentists reported that they would biopsy suspicious lesions. The opinions of respondents regarding oral cancer are given in table 4 . Seventy-nine (51.6\%) dentists felt that their knowledge about oral cancer is current and 88 (57.5\%) felt that they had adequate training to examine patients for oral cancer. Fifty-nine (38.6\%) felt confident to diagnose oral cancer.
Further analysis also showed that dentists who judged themselves as confident in diagnosing oral cancer scored marginally higher in risk factor $(9.1 \pm 2.0)$ and diagnostic knowledge $(12 \pm 2.4)$ as compared to those who were not self-confident $(8.6 \pm 2.4$ and $11.7 \pm 2.4$, respectively). However, a significant difference $(\mathrm{p}<0.05)$ was noted between the total mean knowledge scores of dentists who attended a recent CE course $(21.6 \pm 3.8)$ and those who did not $(20.2 \pm 4.0)$.

\section{Discussion}

Dentists need to possess a thorough knowledge of risk factors, clinical signs and symptoms of oral cancer in order to be effective in identifying, referring and counseling high-risk patients. In this study, only a quarter of the total participants had a high index on the knowledge of 
Fig. 3. Percent distribution of dentists according to diagnostic practice of oral cancer.

Table 4. Opinions of respondents regarding oral cancer

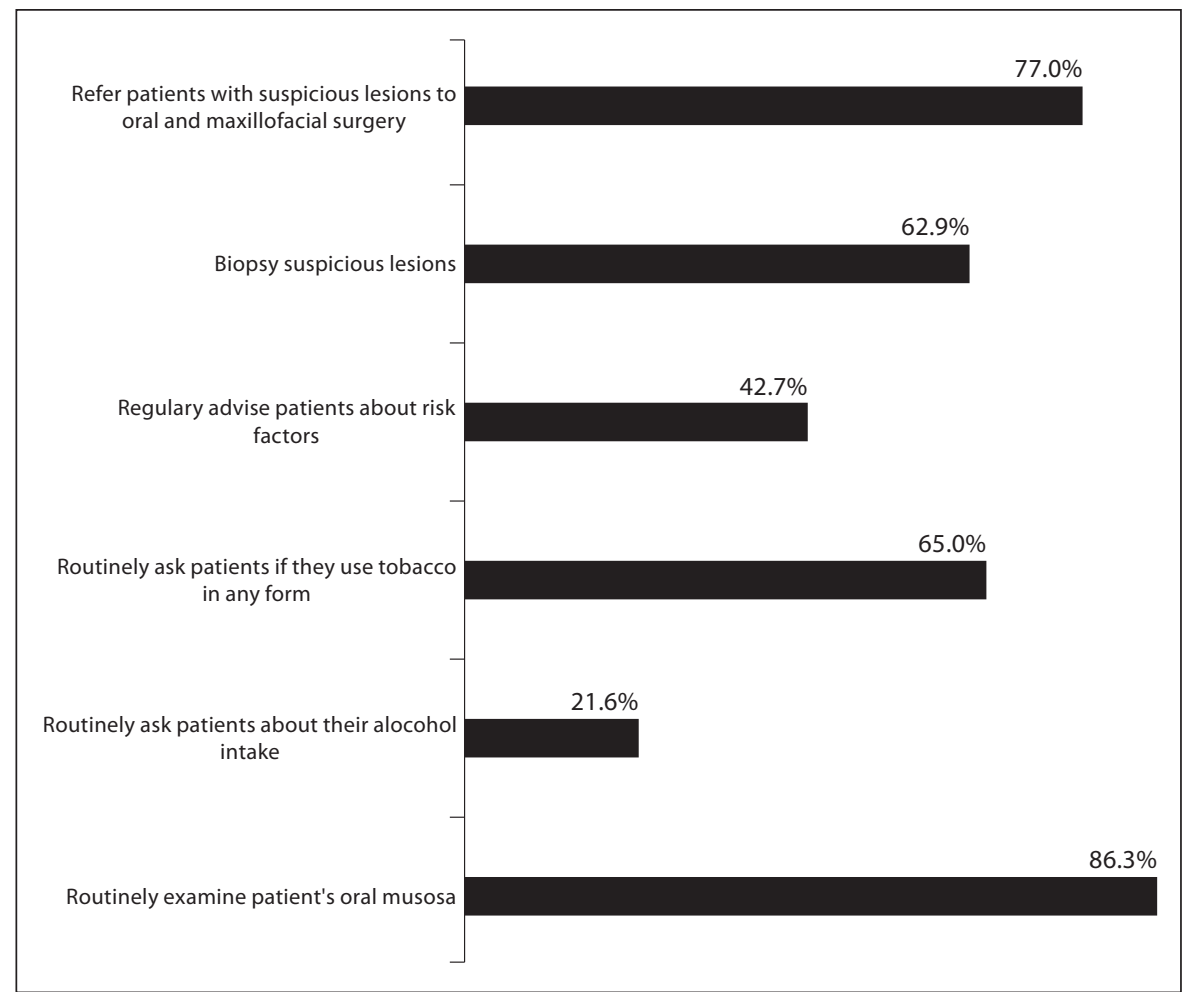

\begin{tabular}{llc}
\hline Opinion & $\mathrm{n}(\%)$ \\
\hline 1 & My knowledge of oral cancer is current & $79(51.6)$ \\
2 & I am comfortable palpating lymph nodes in the neck & $111(72.5)$ \\
3 & I am comfortable referring suspicious oral lesions to specialists & $137(89.5)$ \\
4 & I feel confident to diagnose oral cancer from clinical appearance & $59(38.6)$ \\
5 & Most dentists are adequately trained to examine patients for oral cancer & $49(32)$ \\
6 & Attended a CE course in oral cancer within the past 5 years & $46(30.1)$ \\
7 & I am adequately trained to provide tobacco cessation education & $59(38.6)$ \\
8 & I am adequately trained to provide alcohol counseling & $31(20.3)$ \\
9 & I am adequately trained to examine patients for oral cancer & $88(57.5)$
\end{tabular}

risk factors, while about three fourths had a high index on knowledge of clinical diagnosis.

Tobacco use is the main risk factor for oral cancer [16] and was identified by all the respondents. Smoking among both sexes, youth as well as adults, is considered a serious public health problem in Kuwait [17]. In spite of this knowledge, only $65 \%$ of the dentists routinely asked patients about their tobacco habit and only $38.6 \%$ of dentists felt that they were adequately trained to provide tobacco cessation education. Even in the absence of oral stigmata of tobacco use, dentists should ask about the patient's past and present tobacco use, advise in order to detect new tobacco users and engage in early preventive activities especially with young patients [18].

Alcohol consumption has a synergistic effect along with the use of tobacco in any form. However, the role of alcohol in Kuwait is unclear since alcohol is legally prohibited and only a few individuals declare its use [17]. Dentists have been encouraged to screen and counsel patients about alcohol use [19]. In the present survey, only one fifth of dentists reported that they routinely ask patients about their alcohol intake and had adequate training to provide alcohol counseling. 
While it is encouraging that the large majority of dentists identified most risk factors for oral cancer, they were much less certain about factors that do not pose a risk similar to other surveys $[5,6,8-10,13,14,20]$. As in previous studies, a large proportion of dentists (more than $90 \%$ incorrectly identified family history of oral cancer as a risk factor $[5,6,8,12]$. Only $40.7 \%$ knew that eating spicy foods has no association with oral cancer and $56 \%$ related poor oral hygiene to the incidence of oral cancer. Nearly three fourths of the respondents $(75.5 \%)$ had the misconception that ill-fitting dentures could pose a risk for oral cancer.

Less than half of the dentists advised patients about risk factors regularly which may be related to their own lack of knowledge about risk factors. Previous studies have also shown that dentists are generally uncomfortable in counseling patients on matters such as smoking or alcohol cessation [21-23]. On the contrary, patients have been found to be receptive to dentists' advice on such matters [24]. Therefore, dentists should advocate healthy lifestyle behavior and actively participate in oral cancer prevention emphasizing the importance of risk factors.

Dentists' knowledge of oral cancer diagnosis was found to be better compared to the knowledge of risk factors. The lateral aspect of the tongue and floor of the mouth are at greatest risk of cancer development [25] and $85 \%$ of the respondents recognized these 'high-risk' areas. The majority of the dentists (98\%) knew that early detection improves survival similar to results seen among dentists surveyed in the USA [5], Canada [6] and Germany [13]. Three fourths of the dentists knew that oral cancers are most often diagnosed in the advanced stage in contrast to only $50 \%$ in most of the other surveys $[5,6$, $8,10,20]$. However, only half of the study sample recognized visual inspection as the most effective screening method. Oral visual screening can reduce mortality in high-risk individuals and has the potential to prevent at least 37,000 oral cancer deaths worldwide [26]. In spite of a high level of knowledge of diagnostic concepts, a lack of confidence in conducting a comprehensive oral cancer examination and diagnosis is evident.

It has been reported in previous studies that increased referral and biopsy practices are significantly associated with greater knowledge of oral cancer [5]. In our study, $89.5 \%$ felt the need to refer suspicious oral lesions to specialists. Departments of Oral, Maxillofacial Surgery and Oral Medicine were the most commonly selected points of referral for patients with suspected oral cancer.

Only a small percentage (30.1\%) had attended a recent $\mathrm{CE}$ course on oral cancer during the last 5 years and they demonstrated better knowledge than those who did not attend, which clearly emphasizes the need for a CE course on the topic. The majority of the participants were interested in obtaining further information about oral cancer, most of them preferring an information pack.

The present study clearly showed that the time of graduation and age of the dentists are factors which have quite an impact on their knowledge. Although there was no significant difference, the dentists of a younger age group and recent graduates demonstrated better knowledge. This emphasizes the need for CE on the topic.

Kuwaiti dentists scored significantly higher than nonKuwaiti dentists in the present study. On further analysis, it was found that the majority of the Kuwaiti dentists $(\mathrm{n}=44 ; 91.7 \%)$ in the sample graduated within 15 years, while only 46 (43.8\%) of non-Kuwaiti dentists were recent graduates. The difference was highly significant $(\mathrm{p}<$ 0.001 ). Hence, the time of graduation might have influenced their knowledge. This further reiterates the need for updating information through CE courses.

A possible limitation of the study was that private dental practitioners were not included in the survey. The majority of the population prefers government dental centers, as the services offered are free. Hence the dentists in the government centers are more likely to examine a wide range of patients. Therefore, the sample for the present study included them rather than the private dentists.

Educational strategies should be aimed at providing current information on risk factors, oral examinations, adjunctive diagnostic aids including biopsy and frequent patient referrals, thus facilitating early detection. It appears to be necessary to emphasize the importance of oral soft tissue examination in the undergraduate curriculum and provide opportunities for dental practitioners to ensure that such examinations become a routine practice and arrive at an accurate diagnosis. In addition to providing information in booklets and digital videodisks, diagnostic evaluation of oral lesions and palpation of lymph nodes in patients, as well as demonstration of biopsy techniques are recommended which are bound to have the most immediate applicability to clinical practice.

\section{Conclusion}

This study highlighted the importance of improved educational methods for dental practitioners on oral cancer detection and premalignant lesions of the oral cavity because the role of dental practitioners in the prevention and early detection of oral cancer is of utmost importance. 


\section{References}

-1 Johnson NW, Warnakulasuriya S, Gupta PC, Dimba E, Chindia M, Otoh EC, Sankaranarayanan R, Califano J, Kowalski L: Global oral health inequalities in incidence and outcomes of oral cancer: causes and solutions. Adv Dent Res 2011;23:237-246.

2 Ferlay J, Shin HR, Bray F, Forman D, Mathers C, Parkin DM: GLOBOCAN 2008, Cancer Incidence and Mortality Worldwide: IARC CancerBase No 10. Lyon, International Agency for Research on Cancer, 2010. http:// globocan.iarc.fr.

3 Joseph BK: Oral cancer: prevention and de tection. Med Princ Pract 2002;11:32-35.

4 Schnetler JFC: Oral cancer diagnosis and delays in referral. Br J Oral Maxillofac Surg 1992;30:210-213.

5 Patton LL, Elter JR, Southerland JH, Strauss RP: Knowledge of oral cancer risk factors and diagnostic concepts among North Carolina dentists: implications for diagnosis and referral. J Am Dent Assoc 2005;136:602-610.

6 Clovis JB, Horowitz AM, Poel DH: Oral and pharyngeal cancer: knowledge and opinions of dentists in British Columbia and Nova Scotia. J Can Dent Assoc 2002;68:415-420.

$\checkmark 7$ Seoane J, Warnakulasuriya S, Varela-Centelles P, Esparza G, Dios PD: Oral cancer: experiences and diagnostic abilities elicited by dentists in North-western Spain. Oral Dis 2006;12:487-492.

8 Yellowitz JA, Horowitz AM, Drury TF, Goodman HS: Survey of US dentists' knowledge and opinions about oral pharyngeal cancer. J Am Dent Assoc 2000;131:653-661.

$\checkmark 9$ López-Jornet P, Camacho-Alonso F, MolinaMiñano F: Knowledge and attitudes about oral cancer among dentists in Spain. J Eval Clin Pract 2010;16:129-133.
10 Alonge OK, Narendran S: Oral cancer knowledge and practices of dentists along the Texas-Mexico border. J Cancer Educ 2004;19:6-11.

11 Gajendra S, Cruz GD, Kumar JV: Oral cancer prevention and early detection: knowledge, practices and opinions of oral health care providers in New York state. J Cancer Educ 2006;21:157-162.

12 Carter LM, Ogden GR: Oral cancer awareness of general medical and general dental practitioners. Br Dent J 2007;203:E10.

13 Hertrampf K, Wiltfang J, Koller M, Klosa K, Wenz HJ: Dentists' perspectives on oral cancer: a survey in Northern Germany and a comparison with international data. Eur J Cancer Prev 2010;19:144-152.

14 Colella G, Gaeta GM, Moscariello A, Angelillo IF: Oral cancer and dentists: knowledge, attitudes, and practices in Italy. Oral Oncol 2008;44:393-399.

15 Jaber L, Shaban S, Hariri D, Smith S: Perceptions of healthcare practitioners in Saudi Arabia regarding their training in oral cancer prevention, and early detection. Int J Health Care Qual Assur 2011;24:8-18.

16 Johnson N: Tobacco use and oral cancer: a global perspective. J Dent Educ 2001;65:328339.

17 Morris RE, Mahmeed BE, Gjorgov AN, Al Jassaf HG, Al Rashid B: The epidemiology of lip, oral cavity and pharyngeal cancers in Kuwait 1979-1988. Br J Oral Maxillofac Surg 2000;38:316-319.

18 Reibel J: Tobacco and oral diseases. Update on the evidence with recommendations. Med Princ Pract 2003;12:22-32.
19 McCann M, Macpherson L, Gibson J: The role of the general dental practitioner in detection and prevention of oral cancer: a review of the literature. Dent Update 2000;27: 404-408.

20 Canto MT, Drury TF, Horowitz AM: Maryland dentists' knowledge of oral cancer risk factors and diagnostic procedures. Health Promot Pract 2001;2:255-262.

21 McCann MF, Macpherson LMD, Binnie VT, Stephen KW: A survey of Scottish primary care dental practitioners' oral cancer-related practices and training requirements. Community Dent Health 2000;17:24-30.

22 Horowitz AM, Drury TF, Canto MT: Practices of Maryland dentists: oral cancer prevention and early detection. Baseline data from 1995. Oral Dis 2000;6:282-288.

23 Cruz GD, Ostroff JS, Kumar JV, Gajendra S: Preventing and detecting oral cancer: oral health care providers' readiness to provide health behavior counseling and oral cancer examinations. J Am Dent Assoc 2000;136: 594-601.

24 Miller PM, Ravenel MC, Shealy AE, Thomas S: Alcohol screening in dental patients: the prevalence of hazardous drinking and patients' attitudes about screening and advice. J Am Dent Assoc 2006;137:1692-1698.

25 Neville BW, Day TA: Oral cancer and precancerous lesions. CA Cancer J Clin 2002;52: 195-215.

26 Sankaranarayanan R, Ramadas K, Thomas G, Muwonge R, Thara S, Mathew B, Rajan B, Trivandrum Oral Cancer Screening Study Group: Effect of screening on oral cancer mortality in Kerala, India: a cluster-randomised controlled trial. Lancet 2005;365: 1927-1933 